\section{Bevacizumab gegen das Ovarialkarzinom: Nutzen vor allem bei Hochrisiko-Erkrankung}

\author{
Der gegen VEGF gerichtete Antikörper Bevacizumab hat die Therapie \\ des fortgeschrittenen Ovarialkarzimoms bereichert. Vor allem sein an \\ der Prognose orientierter Einsatz scheint Erfolg versprechend.
}

$V^{2}$ or der Einführung zielgerichteter Antikörper wie Bevacizumab unterzogen sich Frauen mit niedriggradigem Ovarialkarzinom in fortgeschrittenem Stadium oder mit schlechter Prognose standardgemäß einer DebulkingOperation mit folgender Carboplatin/Paclitaxel-Behandlung. Die zusätzliche Bevacizumab-Therapie verlängert im Erstlinien-Setting das progressionsfreie Überleben (PFS) dieser Frauen signifikant - bestätigt u. a. in den zwei großen, randomisierten Phase-III-Studien GOG218 [Burger RA et al. N Engl J Med. 2011; 365(26):2473-83] und ICON7 [Perren TJ et al. N Engl J Med. 2011; 365(26):248496]. Auch im Rezidiv verzögert die VEGF-Blockade den Progress. Die Daten dieser Studien führten zur Zulassung von Bevacizumab für die Erst- und Zweitlinientherapie des fortgeschrittenen Ovarialkarzinoms.

Nun geben die reifen Langzeitdaten der ICON7-Studie Aufschluss, für welche Frauen die Antikörper-Zusatztherapie besonders sinnvoll ist. Einbezogen waren 1.528 Patientinnen nach der Operation - entweder im frühen Erkrankungsstadium mit hohem Risiko (FIGO-Stadium I/IIa bei Grad-3- oder Klarzellen-Histologie) oder im fortgeschrittenen Stadium (FIGO-Stadium IIb-IV). Beim 3-Jahres-PFS, dem primären Endpunkt, hatten Patientinnen unter Standardchemotherapie plus Bevacizumab signifikant besser abgeschnitten als die Frauen unter der Carboplatin/Paclitaxel-Kombination alleine (21,3 vs. 20,3 Monate; Hazard Ratio [HR] 0,81, $95 \%$-Konfidenzintervall [95\%-KI] $0,70-0,94 ; \mathrm{p}=0,004)$. Besonders effektiv war die VEGF-Blockade bei Frauen mit einem hohen Progressionsrisiko. In dieser Subgruppe wurden Patientinnen mit Stadium FIGO IV und FIGO III mit Tumorrest $>1 \mathrm{~cm}$ zusammengefasst.

Median betrug die Gesamtbeobachtungszeit 48,9 Monate. Die Langzeitda- ten ergaben insgesamt keinen statistisch signifikanten Unterschied im Gesamtüberleben zwischen den beiden Behandlungsarmen. Patientinnen der Hochrisikogruppe lebten im Vergleich zu Patientinnen der Niedrigrisikogruppe - mit Stadium FIGO III und einem Tumorrest $<1 \mathrm{~cm}$ oder ohne Tumorrest - durch die Bevacizumab-Addition jedoch signifikant länger (39,7 vs. 34,5 Monate; $\mathrm{p}=0,03)$.

Fazit: Der gegen VEGF gerichtete Antikörper Bevacizumab scheint insbesondere bei Ovarialtumoren mit hohem Progressionsrisiko, z. B. bei großen postoperativen Residualtumoren, im Vergleich zu einer alleinigen Chemotherapie die Gesamtüberlebenszeit zu verlängern. In weiteren Studien sollten nun die Vorteile der Antiangiogenese speziell bei dieser Hochrisiko-Konstellation untersucht werden. Barbara Kreutzkamp

Oza AM et al. Standard chemotherapy with or without bevacizumab for women with newly diagnosed ovarian cancer (ICON7): overall survival results of a phase 3 randomised trial. Lancet Oncol. 2015;16(8):928-36.

\section{Kommentar von Prof. Schmalfeldt:}

Gemäß der S3 Leitlinie „Diagnostik, Therapie und Nachsorge Maligner Ovarialtumoren“ (AWMF-Registernummer: 032/035OL) kann die zusätzliche Behandlung mit Bevacizumab in der Primärtherapie des fortgeschrittenen Ovarialkarzinoms im Stadium FIGO IIIB-IV erwogen werden. Diese Empfehlung resultiert aus den Daten der Sudien GOG-218 und ICON7, in denen die Hinzunahme von Bevacizumab in der Primärtherapie des fortgeschrittenen Ovarialkarzinoms untersucht wurde.

In beiden Studien zeigte sich, dass durch die zusätzliche Angiogenesehemmung das PFS der Patientinnen signifikant verlängert wird. Während in die GOG-Studie überwiegend Patientinnen mit einem postoperativen Tumorrest von mehr als $1 \mathrm{~cm}$ rekrutiert wurden, hatten in der ICON7-Studie zwei Drittel der Patientinnen einen Tumorrest kleiner $1 \mathrm{~cm}$.

Aktuell wurden nun aus der ICON7-Studie die Langzeitdaten zum Überleben publiziert. Hinsichtlich der Nebenwirkungen trat lediglich ein weiteres Grad-3-Ereignis - in Form einer gastrointestinalen Fistel - im Bevacizumab-Arm auf.

Für die Gesamtpopulation konnte kein Vorteil im Gesamtüberleben durch die Hinzunahme von Bevacizumab nachgewiesen werden. Der Unterschied bezüglich des PFS war numerisch gleich zur Voranalyse, aber nicht mehr signifikant.

Patientinnen mit forgeschrittenem Ovarialkarzinom und postoperativ $>1 \mathrm{~cm}$ Tumorrest profitieren am meisten von Bevacizumab.

Interessant und klinisch bedeutsam sind die Ergebnisse in den vor Studienbeginn definierten Subgruppen. Die signifikante Verlängerung des medianen Gesamtüberlebens durch die Bevacizumab-Addition in der Gruppe der Hochrisikopatientinnen ist beachtlich. Dieser Unterschied im Gesamtüberleben blieb über das Intervall der Bevacizumab-Gabe von 12 Monaten hinaus erhalten. Auch 36 Monate nach Randomisation bestand ein Unterschied von $10 \%$ in der Überlebensrate zwischen beiden Gruppen.

In der aktuellen Analyse wurde zusätzlich der Nutzen der Bevacizumab-Gabe in Abhängigkeit von Operationsergebnis und Stadium untersucht. Hierbei zeigte sich kein Vorteil der Antikörpergabe bei Patientinnen mit frühem Ovarialkarzinom und Patientinnen, die tumorfrei operiert werden konnten. Bei diesen Ergebnissen handelt es ich um nicht geplante Subgruppenanalysen; trotzdem bleibt als Fazit festzuhalten, dass Patientinnen mit fortgeschrittenen Ovarialkarzinomen und Tumorrest $>1 \mathrm{~cm}$ nach Primäroperation am meisten von der Bevacizumab-Gabe profitieren.

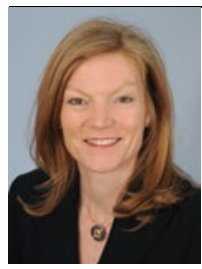

Prof. Dr. med Barbara Schmalfeldt Direktorin der Klinik und Poliklinik für Gynäkologie, Universitätsklinikum Hamburg Eppendorf b.schmalfeldt@uke.de 